\title{
Dirac Sea Effects on Superfluidity in Nuclear Matter
}

\author{
Masayuki Matsuzaki * \\ Department of Physics, Fukuoka University of Education, Munakata, Fukuoka 811-4192, Japan
}

(April 16, 2018)

\begin{abstract}
We study two kinds of Dirac sea effects on the ${ }^{1} S_{0}$ pairing gap in nuclear matter based on the relativistic Hartree approximation to quantum hadrodynamics and the Gor'kov formalism. We show that the vacuum fluctuation effect on the nucleon effective mass is more important than the direct coupling between the Fermi sea and the Dirac sea due to the pairing interaction. The effects of the high-momentum cutoff are also discussed.
\end{abstract}

*Electronic address: matsuza@fukuoka-edu.ac.jp 
Superfluidity caused by the pairing correlation between two nucleons with the linear or angular momenta opposite to each other is a key ingredient to describe quantitatively the thermal evolution of neutron stars and the structure of finite nuclei. As a way of description, relativistic models are attracting attention. The origin of relativistic nuclear models can be traced back to the work of Duerr [1]. Since Chin and Walecka succeeded in reproducing the saturation property of symmetric nuclear matter within the mean-field theory (MFT) with the no-sea approximation [2], quantum hadrodynamics (QHD) has described the bulk properties not only of infinite matter but of finite spherical, deformed and rotating nuclei successfully [3,4]. These successes indicate that the particle-hole (p-h) interaction in QHD is realistic. However, various observables of nuclear many-body systems are sensitive to the single-particle properties around the Fermi surface. The knowledge of the residual particleparticle (p-p) interaction is required to describe them. Since this is still less understood, non-relativistic interactions such as Gogny force are used in the pairing channel in practical "relativistic" MFT calculations.

The first study of a relativistic p-p interaction for the pairing channel in the nuclear medium was done by Kucharek and Ring [5]. They adopted, as the particle-particle interaction $\left(v_{\mathrm{pp}}\right)$ in the gap equation, a one-boson-exchange (OBE) interaction with the ordinary relativistic MFT parameters which gave the saturation under the no-sea approximation. The resulting maximum gap was about three times larger than the accepted values in the non-relativistic calculations [6,7,8,9, 10,11]. Various modifications to improve this result were proposed. They can be classified into two groups: One is to adopt the $v_{\text {pp }}$ which is consistent with the p-h channel [12, 13, 14], and the other is to adopt effective ones which are not explicitly consistent with the p-h channel [15, 3, 16]. Among the former, we examined the $\mathrm{p}$-h polarization in $v_{\mathrm{pp}}$ which reduced the pairing gap in the non-relativistic models [17,18]. But the result was negative; this suggests that the nucleon-antinucleon $(\mathrm{N}-\overline{\mathrm{N}})$ polarization should be taken into account simultaneously [14]. Before doing this, the antinucleon degrees of freedom have to be taken into account in the OBE step as did by Guimarães et al [12]. In this paper, we discuss a more important effect of the Dirac sea than that they discussed.

Our formulation consists of three steps. In the first step, the equations of motion of the normal and the anomalous Green's functions, $G$ and $F$, are derived. We start from the ordinary $\sigma-\omega$ model Lagrangian density,

$$
\begin{aligned}
& \mathcal{L}=\bar{\psi}\left(i \gamma_{\mu} \partial^{\mu}-M\right) \psi \\
& \quad+\frac{1}{2}\left(\partial_{\mu} \sigma\right)\left(\partial^{\mu} \sigma\right)-\frac{1}{2} m_{\sigma}^{2} \sigma^{2}-\frac{1}{4} \Omega_{\mu \nu} \Omega^{\mu \nu}+\frac{1}{2} m_{\omega}^{2} \omega_{\mu} \omega^{\mu}+g_{\sigma} \bar{\psi} \sigma \psi-g_{\omega} \bar{\psi} \gamma_{\mu} \omega^{\mu} \psi \\
& \Omega_{\mu \nu}=\partial_{\mu} \omega_{\nu}-\partial_{\nu} \omega_{\mu} .
\end{aligned}
$$

The Hamiltonian density $\mathcal{H}$ is derived from $\mathcal{L}$ and the chemical potential $\mu$ is introduced in the Hamiltonian, $H^{\prime}=\int\left(\mathcal{H}-\mu \psi^{\dagger} \psi\right) d^{3} x$. The equations of motion for

$$
\begin{aligned}
& G_{\alpha \beta}\left(x-x^{\prime}\right)=-i\left\langle\tilde{0}\left|T \psi_{\alpha}(x) \bar{\psi}_{\beta}\left(x^{\prime}\right)\right| \tilde{0}\right\rangle, \\
& F_{\alpha \beta}\left(x-x^{\prime}\right)=-i\left\langle\tilde{0}\left|T \bar{\psi}_{\alpha}(x) \bar{\psi}_{\beta}\left(x^{\prime}\right)\right| \tilde{0}\right\rangle,
\end{aligned}
$$

are derived from $i \partial_{t} \psi=\left[\psi, H^{\prime}\right]$. Here we note that the Green's functions have to be defined by the superfluid ground state $|\tilde{0}\rangle$ in order to introduce the antinucleon in the next step. Aside from this, this first step is essentially the same as the formulation of Ref. [5]. After 
evaluating the commutator with the interaction term in $H^{\prime}$, the meson fields are eliminated by using the inverse of the Klein-Gordon equations. Here the gauge term in the propagator of the $\omega$ meson can be discarded since $\omega$ couples to the baryon current which is conserved in average in the present superfluid case. The result is

$$
\begin{aligned}
& \left(\not p-M+\gamma^{0} \mu\right)_{\alpha \gamma} G_{\gamma \beta}\left(x-x^{\prime}\right)=\delta_{\alpha \beta} \delta^{4}\left(x-x^{\prime}\right)+i V_{\alpha \gamma, \delta \epsilon}\left\langle\tilde{0}\left|T \psi_{\epsilon}(t, \mathbf{y}) \bar{\psi}_{\delta}(t, \mathbf{y}) \psi_{\gamma}(x) \bar{\psi}_{\beta}\left(x^{\prime}\right)\right| \tilde{0}\right\rangle \\
& \left(-\not{p}-M+\gamma^{0} \mu\right)_{\gamma \alpha} F_{\gamma \beta}\left(x-x^{\prime}\right)=i V_{\gamma \alpha, \delta \epsilon}\left\langle\tilde{0}\left|T \psi_{\epsilon}(t, \mathbf{y}) \bar{\psi}_{\delta}(t, \mathbf{y}) \bar{\psi}_{\gamma}(x) \bar{\psi}_{\beta}\left(x^{\prime}\right)\right| \tilde{0}\right\rangle \\
& V_{\alpha \gamma, \delta \epsilon}=-g_{\sigma}^{2} D_{\sigma} \delta_{\alpha \gamma} \delta_{\delta \epsilon}+g_{\omega}^{2} D_{\omega}\left(\gamma^{\mu}\right)_{\alpha \gamma}\left(\gamma_{\mu}\right)_{\delta \epsilon} \\
& D_{i}=\frac{1}{-\triangle+m_{i}^{2}}, \quad(i=\sigma, \omega)
\end{aligned}
$$

with $x=(t, \mathbf{x})$ and $\not p=i \beta\left(\partial_{t}-\alpha \cdot \nabla\right)$. Here we introduced an instantaneous approximation since it was reported in the preceeding works [12, 13] that the retardation effects are small. The time-ordered products in the right-hand side are decomposed à la Gor'kov [19] by extending Wick's theorem,

$$
\begin{aligned}
& \left\langle\tilde{0}\left|T \psi_{\epsilon}(t, \mathbf{y}) \bar{\psi}_{\delta}(t, \mathbf{y}) \psi_{\gamma}(x) \bar{\psi}_{\beta}\left(x^{\prime}\right)\right| \tilde{0}\right\rangle \\
& =-\rho_{\epsilon \delta^{\prime}}(0) \gamma_{\delta^{\prime} \delta}^{0} i G_{\gamma \beta}\left(x-x^{\prime}\right)+\rho_{\gamma \delta^{\prime}}(\mathbf{x}-\mathbf{y}) \gamma_{\delta^{\prime} \delta}^{0} i G_{\epsilon \beta}\left(y-x^{\prime}\right)-\kappa_{\gamma \epsilon}(\mathbf{x}-\mathbf{y}) i F_{\delta \beta}\left(y-x^{\prime}\right), \\
& \left\langle\tilde{0}\left|T \psi_{\epsilon}(t, \mathbf{y}) \bar{\psi}_{\delta}(t, \mathbf{y}) \bar{\psi}_{\gamma}(x) \bar{\psi}_{\beta}\left(x^{\prime}\right)\right| \tilde{0}\right\rangle \\
& =-\rho_{\epsilon \delta^{\prime}}(0) \gamma_{\delta^{\prime} \delta}^{0} i F_{\gamma \beta}\left(x-x^{\prime}\right)+\rho_{\epsilon \gamma^{\prime}}(\mathbf{y}-\mathbf{x}) \gamma_{\gamma^{\prime} \gamma}^{0} i F_{\delta \beta}\left(y-x^{\prime}\right) \\
& +\kappa_{\delta^{\prime} \gamma^{\prime}}^{*}(\mathbf{y}-\mathbf{x}) \gamma_{\delta^{\prime} \delta}^{0} \gamma_{\gamma^{\prime} \gamma}^{0} i G_{\epsilon \beta}\left(y-x^{\prime}\right),
\end{aligned}
$$

where the normal and the anomalous densities are

$$
\begin{aligned}
& \rho_{\alpha \beta}(\mathbf{x}-\mathbf{y})=\left\langle\tilde{0}\left|\psi_{\beta}^{\dagger}(t, \mathbf{y}) \psi_{\alpha}(x)\right| \tilde{0}\right\rangle \\
& \kappa_{\alpha \beta}(\mathbf{x}-\mathbf{y})=\left\langle\tilde{0}\left|\psi_{\beta}(t, \mathbf{y}) \psi_{\alpha}(x)\right| \tilde{0}\right\rangle .
\end{aligned}
$$

The selfenergy and the pairing field are defined by these densities as

$$
\begin{aligned}
& \Sigma_{\alpha \gamma}(\mathbf{x}-\mathbf{y})=\left(V_{\alpha \gamma, \delta \epsilon} \rho_{\epsilon \delta^{\prime}}(0)-V_{\alpha \epsilon, \delta \gamma} \rho_{\epsilon \delta^{\prime}}(\mathbf{x}-\mathbf{y})\right) \gamma_{\delta^{\prime} \delta}^{0} \\
& \Delta_{\alpha \gamma}(\mathbf{x}-\mathbf{y})=\gamma_{\alpha \alpha^{\prime}}^{0} \gamma_{\gamma \gamma^{\prime}}^{0} V_{\alpha^{\prime} \delta, \gamma^{\prime} \epsilon} \kappa_{\delta \epsilon}(\mathbf{x}-\mathbf{y})
\end{aligned}
$$

The Fourier transform of the equations of motion for $g$ and $f$ defined by $G_{\gamma \beta}=g_{\gamma \beta^{\prime}} \gamma_{\beta^{\prime} \beta}^{0}$ and $F_{\gamma \beta}=f_{\gamma^{\prime} \beta^{\prime}} \gamma_{\gamma^{\prime} \gamma}^{0} \gamma_{\beta^{\prime} \beta}^{0}$ is given by

$$
\left(\begin{array}{cc}
(\omega-h+\mu)_{\alpha \gamma} & -\Delta_{\alpha \gamma} \\
\Delta_{\alpha \gamma}^{*} & \left(\omega+h^{*}-\mu\right)_{\alpha \gamma}
\end{array}\right)\left(\begin{array}{c}
g_{\gamma \beta} \\
f_{\gamma \beta}
\end{array}\right)=\left(\begin{array}{c}
\delta_{\alpha \beta} \\
0
\end{array}\right),
$$

where $h=\alpha \cdot \mathbf{k}+\beta(M+\Sigma(k))$.

In the second step, we derive the equations for the Bogoliubov transformation amplitudes by expressing $g$ and $f$ in Eq.(17) in terms of them. We introduce the Dirac field in the Schrödinger picture,

$$
\psi(\mathbf{x})=\frac{1}{\sqrt{V}} \sum_{\lambda=(\mathbf{k} s)}\left(a_{\lambda} U(\lambda) \mathrm{e}^{i \mathbf{k x}}+b_{\lambda}^{\dagger} V(\lambda) \mathrm{e}^{-i \mathbf{k x}}\right),
$$


to manipulate the $t$-dependence. Here the normalization of $U$ and $V$ is chosen conforming to Ref. [20]. The Bogoliubov amplitudes are defined by

$$
\begin{aligned}
& A_{\lambda}=\left\langle\tilde{0}\left|a_{\lambda} \eta_{\lambda}^{\dagger}\right| \tilde{0}\right\rangle, \quad B_{\lambda}=\left\langle\tilde{0}\left|b_{-\lambda}^{\dagger} \eta_{\lambda}^{\dagger}\right| \tilde{0}\right\rangle, \\
& C_{\lambda}=\left\langle\tilde{0}\left|a_{-\lambda}^{\dagger} \eta_{\lambda}^{\dagger}\right| \tilde{0}\right\rangle, \quad D_{\lambda}=\left\langle\tilde{0}\left|b_{\lambda} \eta_{\lambda}^{\dagger}\right| \tilde{0}\right\rangle,
\end{aligned}
$$

as the overlaps between the quasiparticle $\eta_{\lambda}^{\dagger}$,

$$
H^{\prime} \eta_{\lambda}^{\dagger}|\tilde{0}\rangle=\mathcal{E}_{k} \eta_{\lambda}^{\dagger}|\tilde{0}\rangle, \quad H^{\prime}|\tilde{0}\rangle=0
$$

and the nucleon or the antinucleon. These give the explicit expressions of $g$ and $f$,

$$
\begin{aligned}
& g_{\gamma \beta}(\omega, \mathbf{k})=\frac{1}{\omega-\mathcal{E}_{k}+i \epsilon} \sum_{s}\left\{\left|A_{\lambda}\right|^{2} U_{\gamma}(\lambda) U_{\beta}^{*}(\lambda)+\left|B_{\lambda}\right|^{2} V_{\gamma}(-\lambda) V_{\beta}^{*}(-\lambda)\right. \\
& \left.+A_{\lambda} B_{\lambda}^{*} U_{\gamma}(\lambda) V_{\beta}^{*}(-\lambda)+B_{\lambda} A_{\lambda}^{*} V_{\gamma}(-\lambda) U_{\beta}^{*}(\lambda)\right\} \\
& +\frac{1}{\omega+\mathcal{E}_{k}-i \epsilon} \sum_{s}\left\{\left|C_{-\lambda}\right|^{2} U_{\gamma}(\lambda) U_{\beta}^{*}(\lambda)+\left|D_{-\lambda}\right|^{2} V_{\gamma}(-\lambda) V_{\beta}^{*}(-\lambda)\right. \\
& \left.+D_{-\lambda} C_{-\lambda}^{*} U_{\gamma}(\lambda) V_{\beta}^{*}(-\lambda)+C_{-\lambda} D_{-\lambda}^{*} V_{\gamma}(-\lambda) U_{\beta}^{*}(\lambda)\right\}, \\
& f_{\gamma \beta}(\omega, \mathbf{k})=\frac{1}{\omega-\mathcal{E}_{k}+i \epsilon} \sum_{s}\left\{C_{\lambda} A_{\lambda}^{*} U_{\gamma}^{*}(-\lambda) U_{\beta}^{*}(\lambda)+D_{\lambda} B_{\lambda}^{*} V_{\gamma}^{*}(\lambda) V_{\beta}^{*}(-\lambda)\right. \\
& \left.+C_{\lambda} B_{\lambda}^{*} U_{\gamma}^{*}(-\lambda) V_{\beta}^{*}(-\lambda)+D_{\lambda} A_{\lambda}^{*} V_{\gamma}^{*}(\lambda) U_{\beta}^{*}(\lambda)\right\} \\
& +\frac{1}{\omega+\mathcal{E}_{k}-i \epsilon} \sum_{s}\left\{C_{-\lambda} A_{-\lambda}^{*} U_{\gamma}^{*}(-\lambda) U_{\beta}^{*}(\lambda)+D_{-\lambda} B_{-\lambda}^{*} V_{\gamma}^{*}(\lambda) V_{\beta}^{*}(-\lambda)\right. \\
& \left.+D_{-\lambda} A_{-\lambda}^{*} U_{\gamma}^{*}(-\lambda) V_{\beta}^{*}(-\lambda)+C_{-\lambda} B_{-\lambda}^{*} V_{\gamma}^{*}(\lambda) U_{\beta}^{*}(\lambda)\right\},
\end{aligned}
$$

the former describes the normal propagation and the $\Delta N=0$ pairing, and the latter describes the ordinary $\Delta N=2$ pairing. This $\Delta N=0$ pairing appears because Cooper pairs can be formed regardless of the sign of the single-nucleon energy. Substituting Eq.(11) into Eq.(7) and defining the matrix elements of the pairing field $\Delta$ as

$$
\begin{aligned}
& \Delta(\lambda)=\mathrm{e}^{-i \alpha_{+}(\lambda)} U_{\alpha}^{\dagger}(\lambda) \Delta_{\alpha \gamma} T_{\gamma \beta} U_{\beta}(\lambda), \\
& \tilde{\Delta}(\lambda)=\mathrm{e}^{-i \alpha_{-}(-\lambda)} V_{\alpha}^{\dagger}(-\lambda) \Delta_{\alpha \gamma} T_{\gamma \beta} V_{\beta}(-\lambda), \\
& \delta(\lambda)=\mathrm{e}^{-i \alpha_{-}(-\lambda)} U_{\alpha}^{\dagger}(\lambda) \Delta_{\alpha \gamma} T_{\gamma \beta} V_{\beta}(-\lambda),
\end{aligned}
$$

for the Fermi sea pairing, the Dirac sea pairing, and the $\Delta N=0$ pairing, respectively, we obtain the equation of the Bogoliubov amplitudes,

$$
\left(\begin{array}{cccc}
\omega-E_{k}+\mu & 0 & -\Delta(\lambda) & -\delta(\lambda) \\
0 & \omega+E_{k}+\mu & -\delta(\lambda) & -\tilde{\Delta}(\lambda) \\
-\Delta(\lambda) & -\delta(\lambda) & \omega+E_{k}-\mu & 0 \\
-\delta(\lambda) & -\tilde{\Delta}(\lambda) & 0 & \omega-E_{k}-\mu
\end{array}\right)\left(\begin{array}{c}
A_{\lambda} \\
B_{\lambda} \\
C_{\lambda} \\
D_{\lambda}
\end{array}\right)=0
$$

Here we used

$$
\begin{aligned}
& h_{\alpha \gamma} U_{\gamma}(\lambda)=E_{k} U_{\alpha}(\lambda), \quad h_{\alpha \gamma} V_{\gamma}(-\lambda)=-E_{k} V_{\alpha}(-\lambda), \\
& E_{k}=\sqrt{\mathbf{k}^{2}+M^{\star 2}}, \\
& T U(\lambda)=\mathrm{e}^{i \alpha_{+}(\lambda)} U^{*}(-\lambda), \quad T V(-\lambda)=\mathrm{e}^{i \alpha_{-}(-\lambda)} V^{*}(\lambda), \\
& T h^{*}=h T, \quad T \Delta^{*}=\Delta T, \quad T=i \gamma^{1} \gamma^{3} .
\end{aligned}
$$


Note that we adopted notations such that Eq.(13) took the same form as that of Ref. 112 and chose a phase convention such that all the matrix elements were real. Among the eigenvalues,

$$
\omega^{2}=\left(E_{k}^{2}+\mu^{2}+\frac{1}{2}\left(\Delta^{2}+\tilde{\Delta}^{2}\right)+\delta^{2}\right)-\frac{1}{2} \sqrt{\left(4 E_{k} \mu-\Delta^{2}+\tilde{\Delta}^{2}\right)^{2}+4 \delta^{2}\left(4 E_{k}^{2}+(\Delta+\tilde{\Delta})^{2}\right)}
$$

corresponds to the Fermi sea pairing in the decoupling $(\delta \rightarrow 0)$ limit.

In the third step, we express $\kappa$, and subsequently the matrix elements $\Delta(\lambda), \tilde{\Delta}(\lambda)$, and $\delta(\lambda)$, in terms of $A_{\lambda}-D_{\lambda}$. The Fourier transform of $\kappa$ in Eq.(可) is given by

$$
\begin{aligned}
\kappa_{\delta \epsilon}(\omega, \mathbf{k})=2 \pi \delta\left(\omega+\mathcal{E}_{k}\right) \sum_{s} & \left\{A_{-\lambda} C_{-\lambda} U_{\epsilon}(-\lambda) U_{\delta}(\lambda)+B_{-\lambda} D_{-\lambda} V_{\epsilon}(\lambda) V_{\delta}(-\lambda)\right. \\
& \left.+A_{-\lambda} D_{-\lambda} U_{\epsilon}(-\lambda) V_{\delta}(-\lambda)+B_{-\lambda} C_{-\lambda} V_{\epsilon}(\lambda) U_{\delta}(\lambda)\right\} .
\end{aligned}
$$

Substituting this into the Fourier transform of the second equation of (6) gives

$$
\begin{aligned}
& \Delta(\lambda)=-\frac{i}{2} \int \frac{d^{3} p}{(2 \pi)^{3}} \frac{1}{4 E_{k} E_{p}}\left(\frac{-g_{\sigma}^{2}}{|\mathbf{k}-\mathbf{p}|^{2}+m_{\sigma}^{2}}\right. \\
& {\left[\operatorname{Tr}\left\{\left(\not k+M^{\star}\right)\left(\not p+M^{\star}\right)\right\} \mathrm{e}^{i \alpha_{+}(\lambda)} A_{\lambda} C_{\lambda}+\operatorname{Tr}\left\{\left(\not k+M^{\star}\right)\left(\not p-M^{\star}\right)\right\} \mathrm{e}^{i \alpha_{-}(-\lambda)} B_{\lambda} D_{\lambda}\right]} \\
& +\frac{g_{\omega}^{2}}{|\mathbf{k}-\mathbf{p}|^{2}+m_{\omega}^{2}} \\
& \left.\left[\operatorname{Tr}\left\{\left(\not k+M^{\star}\right) \gamma_{\mu}\left(\not p+M^{\star}\right) \gamma_{\mu}\right\} \mathrm{e}^{i \alpha_{+}(\lambda)} A_{\lambda} C_{\lambda}+\operatorname{Tr}\left\{\left(\not k+M^{\star}\right) \gamma_{\mu}\left(\not \not-M^{\star}\right) \gamma_{\mu}\right\} \mathrm{e}^{i \alpha_{-}(-\lambda)} B_{\lambda} D_{\lambda}\right]\right) \text {, } \\
& \tilde{\Delta}(\lambda)=-\frac{i}{2} \int \frac{d^{3} p}{(2 \pi)^{3}} \frac{1}{4 E_{k} E_{p}}\left(\frac{-g_{\sigma}^{2}}{|\mathbf{k}-\mathbf{p}|^{2}+m_{\sigma}^{2}}\right. \\
& {\left[\operatorname{Tr}\left\{\left(\tilde{\not k}-M^{\star}\right)\left(\not p+M^{\star}\right)\right\} \mathrm{e}^{i \alpha_{+}(\lambda)} A_{\lambda} C_{\lambda}+\operatorname{Tr}\left\{\left(\tilde{\not k}-M^{\star}\right)\left(\not p-M^{\star}\right)\right\} \mathrm{e}^{i \alpha_{-}(-\lambda)} B_{\lambda} D_{\lambda}\right]} \\
& +\frac{g_{\omega}^{2}}{|\mathbf{k}-\mathbf{p}|^{2}+m_{\omega}^{2}} \\
& \left.\left[\operatorname{Tr}\left\{\left(\tilde{\not k}-M^{\star}\right) \gamma_{\mu}\left(\not p+M^{\star}\right) \gamma_{\mu}\right\} \mathrm{e}^{i \alpha_{+}(\lambda)} A_{\lambda} C_{\lambda}+\operatorname{Tr}\left\{\left(\tilde{\not k}-M^{\star}\right) \gamma_{\mu}\left(\not \not-M^{\star}\right) \gamma_{\mu}\right\} \mathrm{e}^{i \alpha_{-}(-\lambda)} B_{\lambda} D_{\lambda}\right]\right) \text {, } \\
& \delta(\lambda)=-\frac{i}{2} \int \frac{d^{3} p}{(2 \pi)^{3}} \frac{1}{4 E_{k} E_{p}}\left(\frac{-g_{\sigma}^{2}}{|\mathbf{k}-\mathbf{p}|^{2}+m_{\sigma}^{2}}\right. \\
& {\left[\operatorname{Tr}\left\{\gamma^{5}\left(\not k-M^{\star}\right) \gamma^{0} \gamma^{5}\left(\not p-M^{\star}\right) \gamma^{0}\right\} \mathrm{e}^{i \alpha_{+}(\lambda)} B_{\lambda} C_{\lambda}\right.} \\
& \left.-\operatorname{Tr}\left\{\gamma^{5}\left(\not k-M^{\star}\right) \gamma^{0} \gamma^{5}\left(\not ̋+M^{\star}\right) \gamma^{0}\right\} \mathrm{e}^{i \alpha_{-}(-\lambda)} A_{\lambda} D_{\lambda}\right] \\
& +\frac{g_{\omega}^{2}}{|\mathbf{k}-\mathbf{p}|^{2}+m_{\omega}^{2}} \\
& {\left[\operatorname{Tr}\left\{\gamma^{5}\left(\not k-M^{\star}\right) \gamma^{0} \gamma_{\mu} \gamma^{5}\left(\not p-M^{\star}\right) \gamma^{0} \gamma_{\mu}\right\} \mathrm{e}^{i \alpha_{+}(\lambda)} B_{\lambda} C_{\lambda}\right.} \\
& \left.\left.-\operatorname{Tr}\left\{\gamma^{5}\left(\not k-M^{\star}\right) \gamma^{0} \gamma_{\mu} \gamma^{5}\left(\not p+M^{\star}\right) \gamma^{0} \gamma_{\mu}\right\} \mathrm{e}^{i \alpha_{-}(-\lambda)} A_{\lambda} D_{\lambda}\right]\right) \text {, }
\end{aligned}
$$

where both $\gamma_{\mu}$ in each line are covariant due to the time reversal. Here we used $A_{-\lambda} D_{-\lambda}=$ $-B_{\lambda} C_{\lambda}$, and so on, derived from the definition of $\kappa$, the $s$-independence of $\mathrm{e}^{i \alpha_{+}(\lambda)} A_{\lambda} C_{\lambda}$, and so on, and

$$
\sum_{s} U(\lambda) \bar{U}(\lambda)=\frac{1}{2 E_{k}}\left(\not k+M^{\star}\right), \quad \sum_{s} V(-\lambda) \bar{V}(-\lambda)=\frac{1}{2 E_{k}}\left(\tilde{\not k}-M^{\star}\right),
$$




$$
\begin{aligned}
& \sum_{s} U(\lambda) \bar{V}(-\lambda)=-\frac{1}{2 E_{k}} \gamma^{5}\left(\not k-M^{\star}\right) \gamma^{0}, \quad \sum_{s} V(-\lambda) \bar{U}(\lambda)=\frac{1}{2 E_{k}} \gamma^{5}\left(\tilde{k}+M^{\star}\right) \gamma^{0}, \\
& \tilde{k}=\left(E_{k},-\mathbf{k}\right) .
\end{aligned}
$$

Equations (13) and (17) form a system of selfconsistent equations. The nucleon effective mass $M^{\star}$ is determined by the selfconsistent condition for the scalar part of the selfenergy, the first equation of (6),

$$
\begin{aligned}
M^{\star} & =M-\frac{g_{\sigma}^{2}}{m_{\sigma}^{2}} \frac{\gamma}{2 \pi^{2}} \int_{0}^{\Lambda_{\mathrm{c}}} \frac{M^{\star}}{\sqrt{\mathbf{p}^{2}+M^{\star 2}}} C_{p}^{2} p^{2} d p \\
& +\frac{g_{\sigma}^{2}}{m_{\sigma}^{2}} \frac{1}{\pi^{2}}\left\{M^{\star 3} \ln \left(\frac{M^{\star}}{M}\right)-M^{2}\left(M^{\star}-M\right)-\frac{5}{2} M\left(M^{\star}-M\right)^{2}-\frac{11}{6}\left(M^{\star}-M\right)^{3}\right\}
\end{aligned}
$$

within the relativistic Hartree approximation (RHA) in which the divergence due to the Dirac sea is renormarized using the counter terms

$$
\mathcal{L}_{\mathrm{ct}}=\alpha_{1} \sigma+\frac{1}{2 !} \alpha_{2} \sigma^{2}+\frac{1}{3 !} \alpha_{3} \sigma^{3}+\frac{1}{4 !} \alpha_{4} \sigma^{4}
$$

Hereafter we suppress the argument $s$, and therefore the direction of $\mathbf{k}$ which defines the direction of $s$, of the gaps and the Bogoliubov amplitudes because they affect only the overall sign. The expression of the vacuum contribution in Eq.(19) is taken from the nonsuperfluid case [20]. We will discuss this later. Then the actual task is to solve the coupled equations (13), (17), and (19). If we neglect the vacuum fluctuation contribution in Eq.(19), the system of equations corresponds to that of Ref. [12]. Here we note that we adopt the Hartree approximation with or without the Dirac sea contribution as in Ref. [5] for the no-sea case.

Now we proceed to the numerical results. Parameters used are $M=939 \mathrm{MeV}, m_{\sigma}=550$ $\mathrm{MeV}, m_{\omega}=783 \mathrm{MeV}, g_{\sigma}^{2}=62.89$ for the RHA or 91.64 for the MFT, $g_{\omega}^{2}=79.78$ for the RHA or 136.2 for the MFT [20], and $\gamma=4$ (symmetric nuclear matter). First we look into the relative magnitudes of the gaps $\Delta(k), \tilde{\Delta}(k)$, and $\delta(k)$. Since $A_{k}$ and $C_{k}$ are dominant among those associated with the eigenvalue Eq.(15), $\Delta(k)$ and $\tilde{\Delta}(k)$ are much larger than $\delta(k)$. From the definition of $\kappa$ in Eq.(5), two kinds of terms, one is antisymmetric in spin space and even with respect to the inversion of the momentum, and the other is symmetric and odd, are possible in $\kappa_{\delta \epsilon}(\omega, \mathbf{k})$. If the finite-range effects are neglected, only six terms of the former type are possible as discussed in Ref. [13]. They correspond to scalar, pseudoscalar, and 4-vector terms in $\kappa T$. Among them, the scalar and the time component of the vector are dominant and consequently $\Delta(k)$ and $\tilde{\Delta}(k)$ are determined by these two terms as pointed out in Ref. 13. Basically this applies also to the calculations of Ref. [12 and ours in which the terms of the latter type are also included. In contrast, $\delta(k)$ which measures the $\Delta N=0$ pairing is given by the pseudoscalar and pseudovector terms in $\kappa T$. Since they contain $B_{k} C_{k}$ and $A_{k} D_{k}$, their typical values are the order of $10^{-6} \mathrm{MeV}$ or less both in the RHA and in the MFT. Therefore, as for the magnitude of the $\Delta N=0$ coupling effect on $\Delta(k)$, our calculation does not agree with that of Ref. [12]. The origin of this disagreement is that their Eq.(54) does not have this structure that the different type of products of the Bogoliubov amplitudes appear in $\delta(k)$. The present result indicates that the Fermi sea pairing and the Dirac sea pairing decouple in accuracy of $10^{-7}$. Accordingly, we use the 
expressions for $\delta(k)=0$ hereafter. Since the equations for $A_{k}$ and $C_{k}$ decoupled from $B_{k}$ and $D_{k}$ are equivalent to a gap equation,

$$
\Delta(k)=-\frac{1}{8 \pi^{2}} \int_{0}^{\Lambda_{\mathrm{c}}} \bar{v}_{\mathrm{pp}}(k, p) \frac{\Delta(p)}{\sqrt{\left(E_{p}-E_{k_{\mathrm{F}}}\right)^{2}+\Delta^{2}(p)}} p^{2} d p
$$

where $\bar{v}_{\mathrm{pp}}(k, p)$ is an antisymmetrized matrix element of the adopted p-p interaction with an instantaneous approximation and an integration with respect to the angle between $\mathbf{k}$ and p to project out the $S$-wave component, the numerical task is greatly simpified to solving the coupled equations (19) and (21).

Next we compare the results of the RHA and the MFT obtained by adopting $\Lambda_{\mathrm{c}}=15$ $\mathrm{fm}^{-1}$ which is large enough for the numerical integrations to converge (see Fig. 3). These are presented in Fig. 1. This shows that the Dirac sea contributes to reducing the gap at low density while to enhancing it at high density. This can be understood as follows: Although both $g_{\sigma}$ and $g_{\omega}$ are reduced in the RHA in comparison with the MFT, altogether they act to reduce the repulsion as shown in Fig. 2. Since both the low-momentum attraction and the high-momentum repulsion give positive contributions to $\Delta\left(k_{\mathrm{F}}\right)$ as discussed in Refs. [15,,16], the above-mentioned reduction of the repulsion leads to reduction of $\Delta\left(k_{\mathrm{F}}\right)$ in the RHA. Besides, the low-momentum attraction decreases steeply as density increases especially in the MFT (not shown). This leads to steeper reduction of $\Delta\left(k_{\mathrm{F}}\right)$ at high density in the MFT than in the RHA. Here we note that this RHA calculation was done by assuming the same vacuum fluctuation contribution as the non-superfluid case as mentioned above. We believe that this approximation is practical because the effect of the pairing on $M^{\star}$ is negligible numerically except at very low density. This indicates that the bulk property, $M^{\star}$, affects the Fermi-surface property, superfluidity, whereas the opposite is not true.

Since QHD is an effective theory of hadrons, form factors or cutoffs related to the spatial size of hadrons might be necessary [21]. On the other hand, an important feature of the gap equation is that it has such a form that the short range correlation is involved [22,23,94. This leads to a similar oscillatory behavior of $\Delta(k)$ to that of $\bar{v}_{\mathrm{pp}}\left(k, k_{\mathrm{F}}\right)$ as functions of $k$ [8, 15, 16]. Matera et al. found that their formulation for the pairing gap gave a cutoff [13]. This is interesting in respect that both which are originated from the pairing correlation act to evade the repulsion at high momentum. They reported that the values they obtained were 1.7 $1.9 \mathrm{fm}^{-1}$. These values mean that the repulsive part was completely cut, see Fig.2. Since our formulation does not have any means to choose a cutoff as those of Refs. [5, 12], here we show the dependence of $\Delta\left(k_{\mathrm{F}}\right)$ at $k_{\mathrm{F}}=0.9 \mathrm{fm}^{-1}$, where it becomes maximum, on the cutoff momentum $\Lambda_{\mathrm{c}}$ as a free parameter. The result is presented in Fig. 3. This shows that the MFT result decreases steeply as the cutoff decreases. This can be understood as follows: The difference between the RHA and the MFT in the low-density case in Fig.1 mainly comes from the difference in the magnitude of the high-momentum repulsion. In other words, the contribution of the high-momentum repulsion is more important in the MFT case. Consequently, as the cutoff momentum decreases, the $\Delta\left(k_{\mathrm{F}}\right)$ of the MFT calculation decreases more. The plateaus around $\Lambda_{\mathrm{c}}=2-3 \mathrm{fm}^{-1}$ in the RHA and $\Lambda_{\mathrm{c}}=1-2 \mathrm{fm}^{-1}$ in the MFT are due to the sign change of $v_{\mathrm{pp}}$ around there (see Fig.2). A comparison with the Bonn-B potential [24] which reproduced the maximum pairing gap accepted in the nonrelativistic studies [15,34 is also shown in Fig.2. This indicates that the high-momentum repulsion of the MFT without a cutoff is too strong and the low-momentum repulsion is 
too weak both in the RHA and in the MFT to give reasonable pairing gaps. A possible improvement in the p-p channel would be to take into account the nucleon-antinucleon $(\mathrm{N}-\overline{\mathrm{N}})$ polarization.

To summarize, we have studied two kinds of Dirac sea effects on the pairing gap in nuclear matter. One is the vacuum fluctuation effect on the nucleon effective mass and the other is the direct coupling between the Fermi sea pairing and the Dirac sea pairing. The former is a bulk effect while the latter affects only the Fermi surface. Our calculation indicates that the former is more important. The dependence of $\Delta\left(k_{\mathrm{F}}\right)$ on the high-momentum cutoff was also discussed and the MFT result has been shown to depend more strongly on it than the RHA one.

Discussions with Prof. M. Nakano and Prof. R. Tamagaki are acknowledged. The author also thanks Mr. T. Tanigawa for calculating the Bonn potential. Numerical calculations were done using the computer system of the Information Processing Center, Fukuoka University of Education. 


\section{REFERENCES}

[1] H. -P. Duerr, Phys. Rev. 103, 469 (1956).

[2] S. A. Chin and J. D. Walecka, Phys. Lett. B52, 24 (1974).

[3] P. Ring, Prog. Part. Nucl. Phys. 37, 193 (1996).

[4] B. D. Serot and J. D. Walecka, Int. J. Mod. Phys. E6, 515 (1997).

[5] H. Kucharek and P. Ring, Z. Phys. A339, 23 (1991).

[6] L. Amundsen and E. Østgaard, Nucl. Phys. A437, 487 (1985).

[7] H. Kucharek, P. Ring, P. Schuck, R. Bengtsson and M. Girod, Phys. Lett. B216, 249 (1989).

[8] M. Baldo, J. Cugnon, A. Lejeune and U. Lombardo, Nucl. Phys. A515, 409 (1990).

[9] T. Takatsuka and R. Tamagaki, Prog. Theor. Phys. Suppl. 112, 27 (1993); and references cited therein.

[10] J. M. C. Chen, J. W. Clark, R. D. Davé and V. V. Khodel, Nucl. Phys. A555, 59 (1993).

[11] Ø. Elgarøy, L. Engvik, M. Hjorth-Jensen and E. Osnes, Nucl. Phys. A604, 466 (1996).

[12] F. B. Guimarães, B. V. Carlson and T. Frederico, Phys. Rev. C 54, 2385 (1996).

[13] F. Matera, G. Fabbri and A. Dellafiore, Phys. Rev. C 56, 228 (1997).

[14] M. Matsuzaki and P. Ring, Proc. of the APCTP Workshop on Astro-Hadron Physics in Honor of Mannque Rho's 60th Birthday: Properties of Hadrons in Matter, 25-31 Oct., 1997, Seoul, Korea (World Scientific, Singapore, in press), [e-print nucl-th/9712060].

[15] A. Rummel and P. Ring, preprint (1996).

[16] M. Matsuzaki and T. Tanigawa, submitted to Phys. Lett. B, [e-print nucl-th/9803044.

[17] J. Wambach, T. L. Ainsworth and D. Pines, Nucl. Phys. A555, 128 (1993).

[18] H.-J. Schulze, J. Cugnon, A. Lejeune, M. Baldo and U. Lombardo , Phys. Lett. B375, 1 (1996).

[19] L. P. Gor'kov, Sov. Phys. JETP, 7, 505 (1958).

[20] B. D. Serot and J. D. Walecka, Adv. Nucl. Phys. 16, 1 (1986).

[21] M. Nakano, N. Noda, T. Mitsumori, K. Koide, H. Kouno and A. Hasegawa, Phys. Rev. C 55, 890 (1997).

[22] L. N. Cooper, R. L. Milles and A. M. Sessler, Phys. Rev. 114, 1377 (1959).

[23] T. Marumori, T. Murota, S. Takagi, H. Tanaka and M. Yasuno, Prog. Theor. Phys. 25, 1035 (1961).

[24] R. Machleidt, Adv. Nucl. Phys. 19, 189 (1989). 


\section{FIGURES}

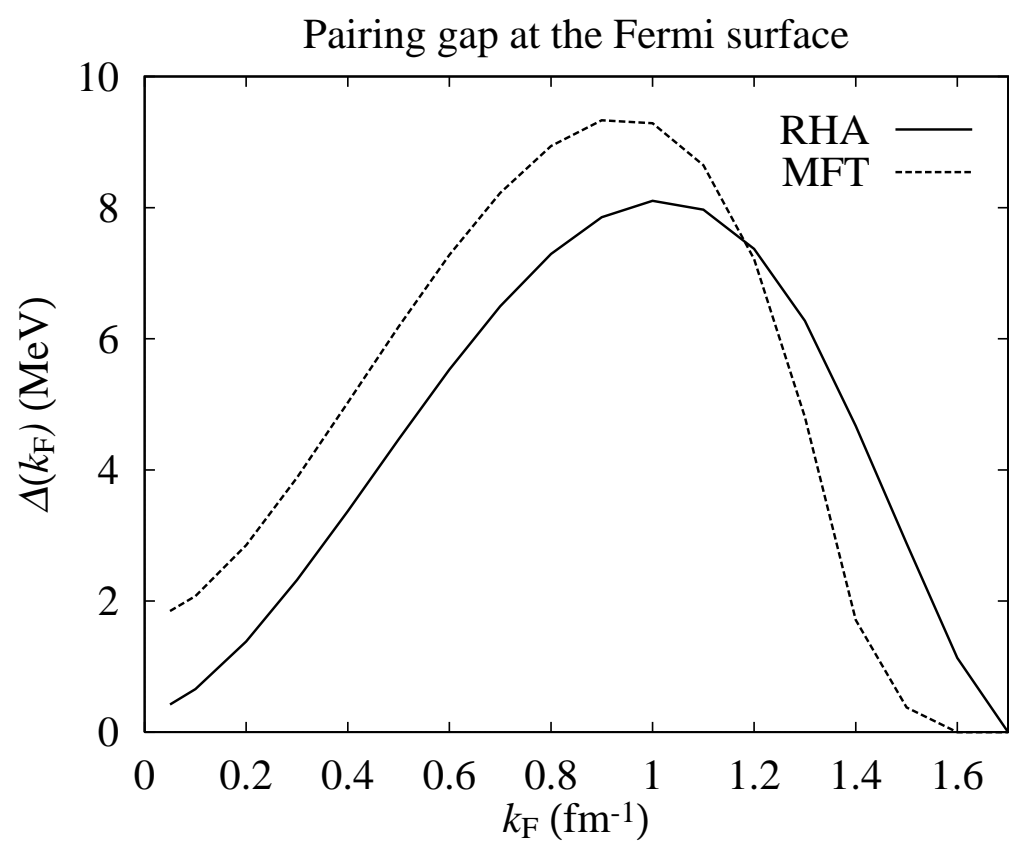

FIG. 1. Pairing gap in symmetric nuclear matter at the Fermi surface as functions of the Fermi momentum. Solid and dashed lines indicate the results obtained by taking and not taking into account the vacuum fluctuation contribution, respectively.

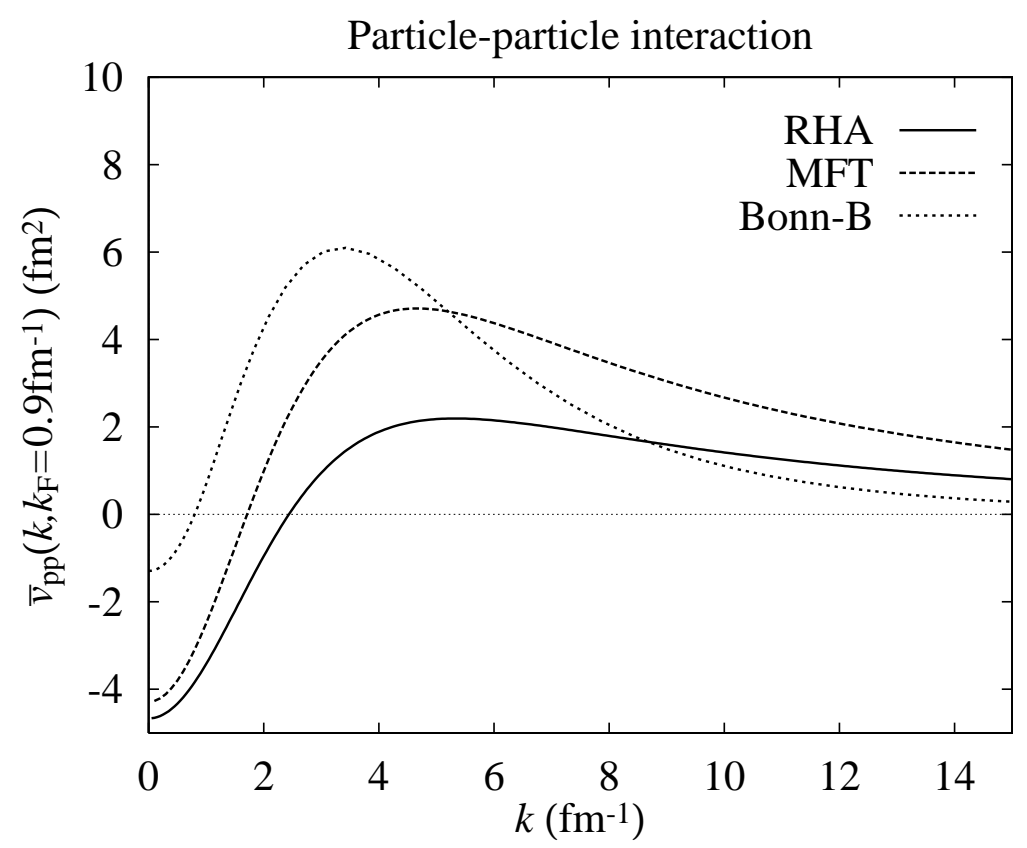

FIG. 2. Matrix element $\bar{v}_{\mathrm{pp}}\left(k, k_{\mathrm{F}}\right)$ as functions of the momentum $k$, with a Fermi momentum $k_{\mathrm{F}}=0.9 \mathrm{fm}^{-1}$. Solid and dashed lines indicate the results obtained by taking and not taking into account the vacuum fluctuation contribution, respectively. The Bonn-B potential for the ${ }^{1} S_{0}$ channel is also shown by the dotted line. 


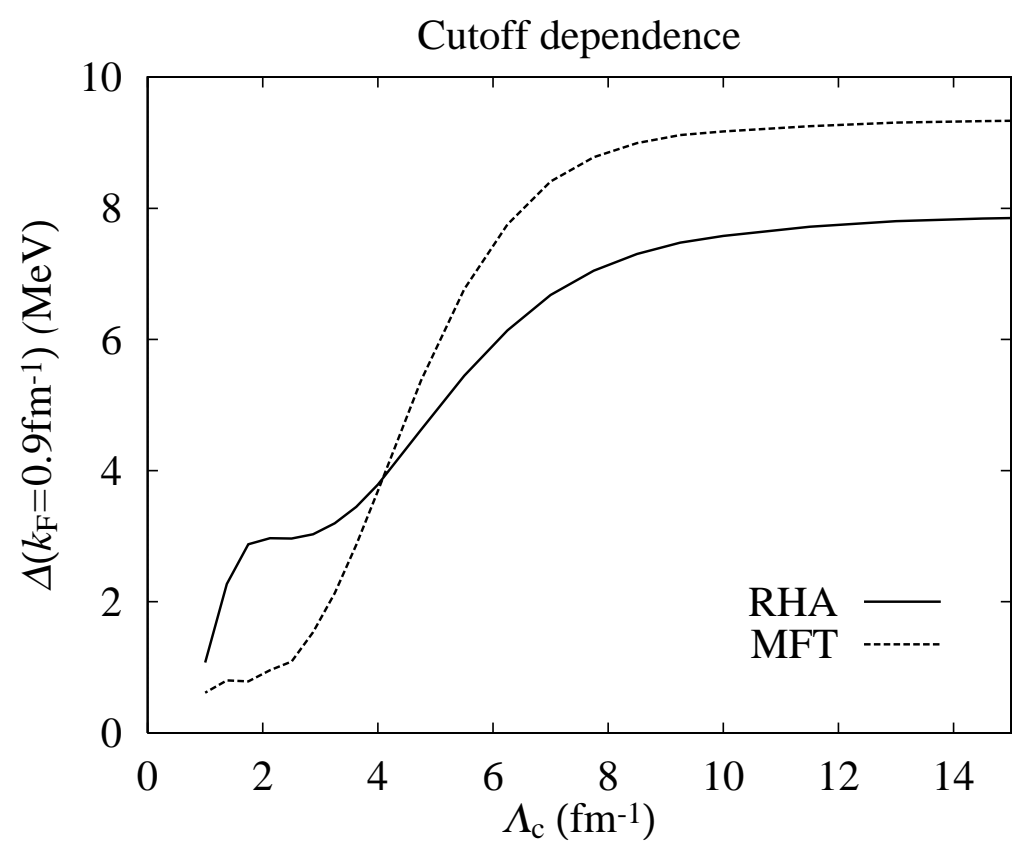

FIG. 3. Pairing gap at the Fermi surface, $k_{\mathrm{F}}=0.9 \mathrm{fm}^{-1}$, as functions of the cutoff parameter in the numerical integrations. Solid and dashed lines indicate the results obtained by taking and not taking into account the vacuum fluctuation contribution, respectively. 